\title{
Hemispheric symmetry in contrast and orientation sensitivity
}

\author{
FREDERICK L. KITTERLE and RUSSELL S. KAYE \\ The University of Toledo, Toledo, Ohio
}

\begin{abstract}
This experiment was designed to determine whether there were hemispheric and/or hemiretinal (nasal/temporal) differences in contrast sensitivity and the oblique effect. Contrast sensitivity functions were measured in the left and right eyes for vertically $\left(90^{\circ}\right)$ and obliquely $\left(45^{\circ}\right)$ oriented sinusoidal gratings presented in the right and left visual fields. There were no hemispheric differences in contrast sensitivity for vertically or obliquely oriented gratings. However, sensitivity was lower for obliquely oriented gratings. Thus, the cerebral hemispheres do not appear to differ in sensitivity to contrast or in the magnitude of the oblique effect. The implications of these results are discussed in terms of the role of spatial frequency channels in information processing asymmetries between the left and right cerebral hemispheres.
\end{abstract}

Many studies indicate that perceptual performance depends upon the visual field in which a stimulus is presented and also upon the nature of the task. For example, orientation matching (Atkinson \& Egeth, 1972; Umiltà et al., 1974), the detection of line curvature (Longden, Ellis, \& Iverson, 1976), the perceptual analysis of letters (Egeth \& Epstein, 1972), the recognition of facial stimuli (Gilbert \& Bakan, 1973), as well as dot enumeration and localization (Kimura, 1969) are performed better when the stimuli are presented in the left visual field (LVF). On the other hand, the conceptual analysis of letters (Egeth \& Epstein, 1972) and the recognition of words (Mishkin \& Forgays, 1952) and digits (Geffen, Bradshaw, \& Nettleton, 1972) have been found to be superior for rightvisual-field (RVF) presentations.

Since the visual pathways arising from the temporal hemiretinas in man pass directly to the ipsilateral cerebral hemisphere and the pathways arising from the nasal hemiretinas project to the contralateral cerebral hemisphere (Davson, 1972), visual stimuli that are presented in the LVF are, initially, represented in the right hemisphere (RH) of the brain. Stimuli presented in the $R V F$, on the other hand, are represented in the left cerebral hemisphere ( $\mathrm{LH})$. The implication of this is that the dependence of performance on visual-field presentation can be assumed to reflect information processing asymmetries between the two cerebral hemispheres.

One of the problems highly debated in the field of cerebral lateralization is whether the asymmetry is present at an early (sensory) stage of information processing or whether it emerges at later (cognitive) stages (see DiLollo, 1981; Moskovitch, 1979; Sergent, 1983a, 1983b).

For example, in light of early research (Campbell \& Robson, 1968) suggesting that visual patterns may be ana-

The authors' mailing address is: Department of Psychology, The University of Toledo, Toledo, Ohio 43606. lyzed in terms of their spatial-frequency Fourier components, there have been recent attempts to account for asymmetries at early stages in the processing of visual information in terms of differences in the sensitivity of the cerebral hemispheres to orientation and spatial frequency (Beaton \& Blakemore, 1981; Rao, Rourke, \& Whitman, 1981; Rose, 1983; Rovamo \& Virsu, 1979; Sergent, 1982a, 1982b, 1982c, 1983a, 1983b; Tei \& Owen, 1980). An important implication of this work is that the nature of the visual input may play an important role in processing asymmetries (Sergent, 1983b). Thus, by varying the spatial frequency content of a target, the advantage one hemisphere has over the other in information processing may be reversed. Indirect support for this hypothesis has been found in studies of perceptual classification (Sergent, 1983a) and face recognition (Sergent, $1982 \mathrm{~b}, 1982 \mathrm{c}$ ).

The RH appears to be specialized for face recognition. Generally, studies have used highly dissimilar faces that are presented briefly. Harmon (1973) and Teiger and Ganz (1979) have shown that low spatial frequencies are sufficient for accurate recognition. As Sergent (1983b) points out, facial recognition may be based upon low spatial frequencies in those studies, either because high spatial frequencies are not present due to low energy or because low spatial frequencies are sufficient for recognition when faces are fairly dissimilar.

The $\mathrm{RH}$ advantage over the $\mathrm{LH}$ in the processing of faces may result from its being better tuned to the low range of spatial frequencies. However, at longer durations and when faces are fairly similar or have well-known physiognomies (Marzi \& Berlucchi, 1977), the LH becomes superior to the $\mathrm{RH}$, because, to reach an accurate decision, the high frequencies contained in the face must now be processed. In this regard, it is interesting to note that most experiments yielding $\mathrm{RH}$ superiority have used black-and-white photographs, which presumably have lower contrast than line drawings and are also presented 
briefly. This may decrease the high-spatial-frequency components and thus favor RH processing. Conversely, experiments with faces showing RVF-LH superiority have used line drawings that yield maximum contrast (Fairweather, Brizzolara, Tabossi, \& Umiltà, 1982; Patterson \& Bradshaw, 1975; Sergent, 1982c) or photographs exposed for a long duration (Jones, 1980; Marzi \& Berlucchi, 1977). Both conditions increase the energy in the high-spatial-frequency range.

A recent study utilizing a paradigm developed by Posner and Mitchell (1967) also supports the view that hemispheric asymmetries in processing may be a function of the spatial frequency content of the target. Sergent (1983b) determined the effect of target size on a perceptual classification task that required subjects to determine whether letters of the same or different case or name matched. Sergent (1983a) found that the RH processed large letters faster than did the LH. Large letters have more low-spatial-frequency components than do small letters, whereas high spatial frequencies are present in both large and small letters. This result suggests sensory limitations in hemispheric processing based upon target spatial frequency. Sergent $(1983 a, 1983 b)$ has concluded that perceptual tasks dependent upon low-spatial-frequency components should impair LH processing more than $\mathrm{RH}$ processing, and that perceptual performance in the $\mathrm{RH}$ should be impaired from reductions in the low-spatial-frequency content of a target.

In addition to the possibility that laterality effects are based upon sensory limitations in the cerebral processing of spatial frequency information, there also is some suggestion that the cerebral hemispheres differ in sensitivity to target orientation (Beaton \& Blakemore, 1981; Fontenot \& Benton, 1972). This may account for RH superiority in orientation matching (Atkinson \& Egeth, 1972; Umiltà et al., 1974) and in the detection of line curvature (Longdon et al., 1976). Two explanations for differences in orientation sensitivity have been put forward (Andrews, 1967). One is that some channels are more sensitive to certain meridians than others. The alternative view is that more channels may subserve certain orientations than others (see Berkley, Kitterle, \& Watkins, 1975).

One demonstration of differential sensitivity to orientation is the "oblique effect"' (Appelle, 1972). This is the finding that sensitivity is generally greater for contours presented in the horizontal and vertical meridians than in the oblique meridians. If performance in orientation matching and line-curvature detection is dependent upon the sensitivity of orientation channels, then the cerebral hemispheres may differ in terms of the magnitude of the oblique effect. The sensitivity to differences in orientation may also be dependent upon the spatial frequency of the stimulus. Berkley et al. (1975) showed that the oblique effect increases with spatial frequency.

Although some studies have interpreted hemispheric asymmetries in terms of sensitivity differences to spatial frequencies, they have not directly measured contrast sen- sitivity for targets presented to each hemisphere as a function of spatial frequency and orientation. The relatively few studies that have measured contrast sensitivity as a function of hemispheric presentation are inconclusive. Although some evidence suggests hemispheric asymmetry (Beaton \& Blakemore, 1981; Rao et al., 1981; Rovamo \& Virsu, 1979), other studies have not been able to replicate these findings (Blake \& Mills, 1979; Rijsdijk, Kroon, $\&$ van der Wildt, 1980; Rose, 1983). Rose (1983) points out that, since only monocular viewing was used, these discrepancies could be due to individual differences in nasotemporal asymmetry (see Rijsdijk et al. 1980). However, although other research has shown that sensitivity is somewhat better in the temporal hemiretinas (Mandelbaum \& Sloan, 1947; Markowitz \& Weitzman, 1969), this cannot alone account for visual field differences (see Fontenot \& Benton, 1972). Nevertheless, retinal sensitivity could interact with hemispheric superiority such that hemispheric superiority and retinal sensitivity could cancel each other, thus abolishing visual-field differences, or differential retinal sensitivity in the absence of hemispheric superiority could create the impression of cerebral asymmetries (Kimura, 1969; Markowitz \& Weitzman, 1969; McKinney, 1967; Overton \& Wiener, 1966).

One of the problems in resolving issues of the level of information processing at which asymmetries arise is that there is a frequent lack of distinction between detection and, for example, discrimination or recognition. Experiments utilizing discrimination or recognition paradigms may involve processing of information at higher levels. Thus, it would be important to determine whether asymmetries between the hemispheres emerge at an early level of processing and are revealed as differences in sensitivity of spatial frequency and/or orientation channels.

The present study addressed these issues. Contrast sensitivity functions were determined with vertical and obliquely oriented gratings for the nasal and temporal retinas of both eyes. Hemispheric asymmetries could be inferred if there was increased contrast sensitivity for the temporal retina of one eye and the nasal retina of the other. Hemiretinal effects, on the other hand, would be reflected in a difference between contrast sensitivity on the nasal and temporal retinas of each eye.

\section{METHOD}

\section{Subjects}

Three male graduate students at the University of Toledo served as observers. They were naive as to the purpose of the experiment and were paid for participation. Each observer had normal or corrected-to-normal vision (minimum of 20/20) in each eye, as determined by a Snellen chart. Each subject was also right-handed (laterality quotient of +.70 or better), as measured by the Edinburgh Handedness Inventory (Oldfield, 1971).

\section{Apparatus and Stimuli}

The stimuli were sinusoidal gratings generated on the face of a Tektronix $5103 \mathrm{D} / \mathrm{N}$ oscilloscope with a P-31 phosphor, using conventional techniques. The stimuli were modulated about the mean 
luminance of the screen, which was $3.2 \mathrm{fL}$, as measured with an SEI photometer. The gratings were presented as $100-\mathrm{msec}$ flashes with a 900 -msec intertrial interval between presentations. Neither changes in spatial frequency nor grating presentation altered the mean luminance of the screen. The contrast levels were calibrated following the method outlined by Mitchell and Wilkinson (1974).

A large matte screen, $32^{\circ} \times 24^{\circ}$, surrounded the display; a hole in the matte screen provided a view of the oscilloscope screen. At the viewing distance of $111.8 \mathrm{~cm}$, the gratings appeared in a circular aperture that subtended $2^{\circ}$. A projector illuminated the screen so that light that matched the oscilloscope in color and brightness fell on the surround but not on the hole. Consequently, in the absence of a grating presentation, the entire screen appeared uniformly illuminated. The oscilloscope was mounted on an assembly that allowed rotation so that the orientation of the gratings could be varied. Small fixation points $\left(10^{\prime}\right)$ placed $2^{\circ}$ to the left and right of the aperture served to place the gratings in either the LVF or the RVF.

A chinrest was used to minimize head movements. Except for the luminance of the screen, the room was dark.

\section{Procedure}

At the outset of the experiment, one of the observer's eyes was patched and his head was placed in the chinrest. The observer adapted to the mean luminance of the screen for $7 \mathrm{~min}$, during which the instructions were read.

Essentially, the experiment involved the determination of the observer's high spatial frequency cutoff (i.e., the maximum number of resolvable cycles of the grating) at each of seven contrast levels $(.02, .03, .06, .13, .22, .31$, and .50$)$ for vertical $\left(90^{\circ}\right)$ and oblique $\left(45^{\circ}\right)$ sinusoidal gratings in central vision and at each fixation point for both the left and the right eye. A method of ascending and descending limits was used. On a given trial, the observer indicated whether the grating was visible; if yes (no) then the spatial frequency was incremented (decremented) until the grating disappeared (appeared). Altogether, there were two ascending and two descending trials in each condition. The grating contrasts were randomized, with orientation, viewing state, and eye counterbalanced across observers. Instructions stressed the importance of maintaining fixation upon the appropriate fixation point. If the observer felt that fixation altered, the trial was repeated. The short presentation time (100 msec) prevented eye movements during stimulus presentation.

The design of the experiment involved three viewing states, two orientations, and seven contrast levels as within-subjects factors for each eye. Four replications in each stimulus condition and five daily sessions produced a total of 20 observations per data point. Each observer had one practice session.

\section{RESULTS}

The results with the vertical gratings are shown in Figures $1 \mathrm{~A}-1 \mathrm{C}$ and with the oblique gratings in Figures 2A-2C for Observers J.S., K.S., and R.D., respectively. Although we measured the highest spatial frequency observable at a number of different contrasts, the data presented in these figures have their axes interchanged and $\log$ contrast sensitivity (reciprocal of contrast) is plotted as a function of log spatial frequency. This method of plotting the data makes it easier to compare the present results with previously published contrast sensitivity functions. The symbols within each figure represent LVF right-eye (circles), LVF left-eye (unfilled triangles), RVF left-eye (filled triangles), and RVF right-eye (squares) data.

The results for the vertical gratings presented in Figure 1 show fairly good agreement among observers.
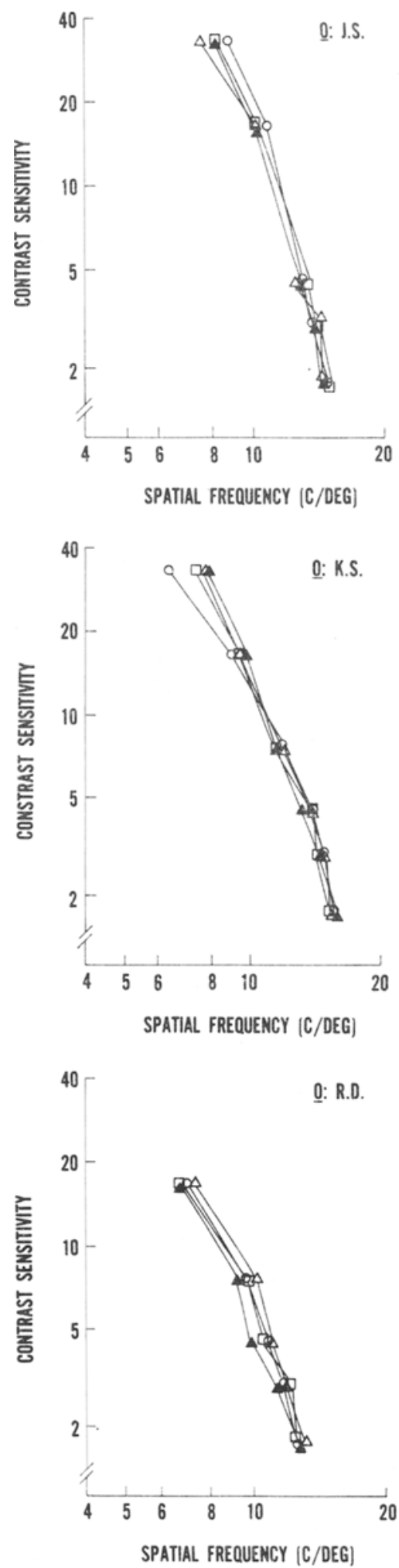

Figure 1. Log contrast sensitivity for detecting a vertical sinusoidal grating plotted as a function of $\log$ spatial frequency. Symbols show the data for: left visual field, right eye (circles); left visual field, left eye (unfilled triangles); right visual field, left eye (filled triangles); and right visual field, right eye (squares). (A) Results for Observer J.S. (B) Results for Observer K.S. (C) Results for Observer R.D. 

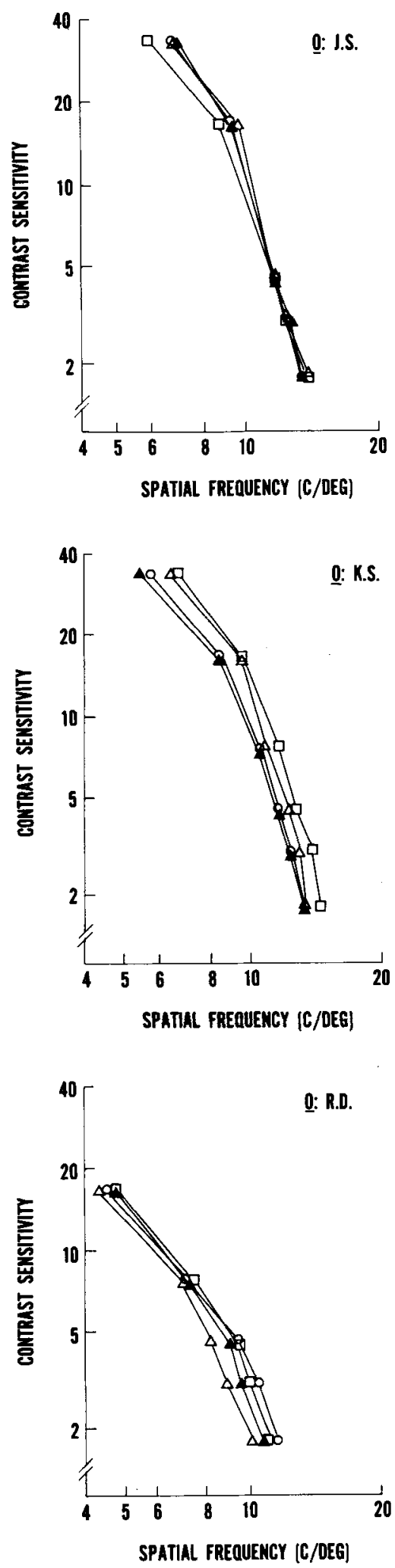

Figure 2. Contrast sensitivity functions for an obliquely oriented grating. Symbols are the same as in Figure 1. (A) Results for Observer J.S. (B) Results for Observer K.S. (C) Results for Observer R.D.
Although it is readily apparent that contrast sensitivity decreases with spatial frequency, there does not appear to be any difference in sensitivity between visual fields or between eyes. Thus, there is no evidence of either hemispheric or hemiretinal differences in contrast sensitivity for vertically oriented gratings.

In Figure 2, a pattern of results obtained with oblique gratings reveals individual differences in the data of Observers K.S. and R.D. For example, at low spatial frequencies, Observer K.S.'s nasal retina appears to be more sensitive than his temporal retina. However, at higher spatial frequencies, there is evidence only for a higher sensitivity of the temporal retina of the left eye. For Observer R.D., on the other hand, contrast sensitivity is somewhat less for the nasal retina of the left eye at high spatial frequencies. It is not clear why a similar pattern of results was not obtained with vertical gratings.

To compare the magnitude of the oblique effect between hemispheres, the data have been replotted in Figures 3A3C. Circles plot the results for vertical gratings presented in the right hemisphere; unfilled triangles plot those in the left hemisphere. Filled triangles plot the results for oblique gratings presented in the right hemisphere, and squares plot the results for the left. It is quite apparent in the data of all observers that the magnitude of the oblique effect is the same for both hemispheres. The somewhat smaller effect at 6 cycles/deg for K.S. is due to differences in sensitivity between the temporal retinas of the left and right eyes.

\section{DISCUSSION}

The purpose of the present study was to determine whether the cerebral hemispheres differed in sensitivity to contrast and in the magnitude of the oblique effect. The results indicate that contrast sensitivity functions measured in the right and left hemispheres are virtually indistinguishable. In addition, the magnitude of the oblique effect appears to be similar in both hemispheres. Thus, information processing asymmetries do not appear to be present in sensitivity to contrast and orientation at threshold levels. However, it might be argued that failure to find hemispheric differences may be due to the insensitivity of the psychophysical method, to the inability of the observers to maintain fixation, or to the high noise levels in the data. In earlier work, we have found that both experienced and naive observers yield considerably less variability when required to determine the cutoff spatial frequency at a number of contrast levels rather than contrast thresholds at different spatial frequencies (Berkley et al., 1975). Consequently, we adopted this method in order to reduce variability. In addition, we used flashed presentations because earlier work (Berkley et al., 1975) had shown that this reduces variability in determining peripheral contrast sensitivity and because relatively brief presentations reduce, if they do not eliminate, the effects of eye movements. If either or both of the above effects were present, a considerably higher degree of variability 

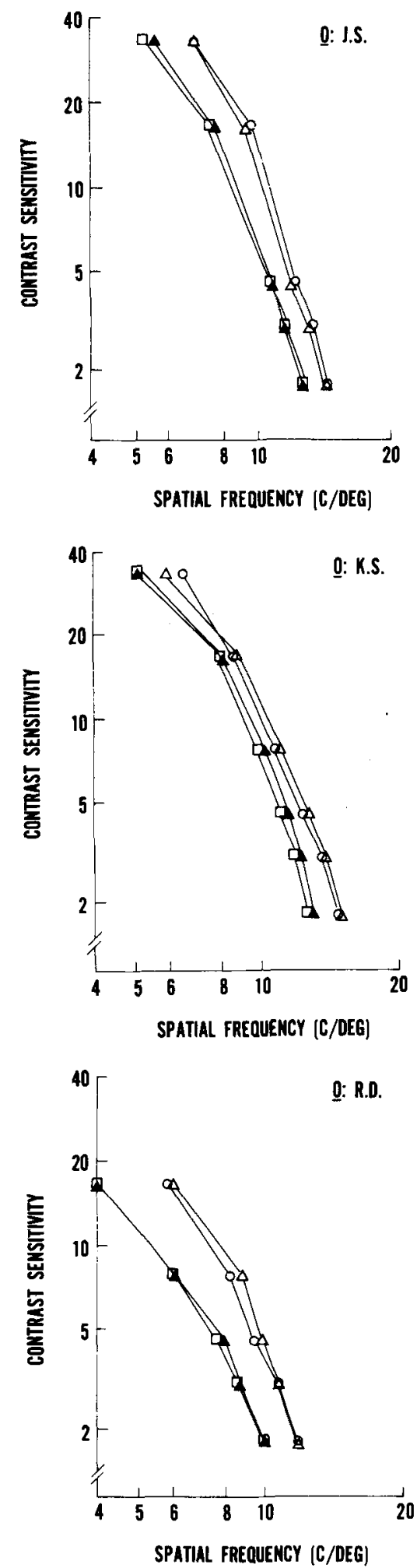

Figure 3. Log contrast sensitivity plotted as a function of log spatial frequency for vertical gratings presented to the right hemisphere (circles) or left hemisphere (unfilled triangles) and for obliquely oriented gratings presented to either the right (filled triangles) or left hemisphere (squares). (A) Results for Observer J.S. (B) Results for Observer K.S. (C) Results for Observer R.D. would have been present in the data. This was not the case-the variability within conditions was extremely low. Thus, the failure to find significant differences between the hemispheres cannot be due to eye-movement artifacts or to the insensitivity of our psychophysical methods.

The findings of the present study differ from those of earlier research, which suggested the existence of laterality effects in contrast sensitivity (Beaton \& Blakemore, 1981; Rao et al., 1981; Rovamo \& Virsu, 1979). It is possible that the earlier results can be attributed to nasaltemporal rather than hemispheric differences in sensitivity, since contrast sensitivity functions were determined with only one eye. K.S.'s and R.D.'s results for obliquely oriented gratings are consistent with this view.

To account for information processing asymmetries in terms of hemispheric rather than nasal-temporal differences, both eyes need to be tested. Hemispheric effects are inferred if differences in contrast sensitivity functions for the LVF and RVF are the same for the right and left eyes. Hemiretinal effects are inferred if visual-field differences for one eye are reversed in the other. As can be seen in the results of the present study when these factors are taken into account, there are very small, but nonsignificant, visual-field differences which are not found consistently across observers or between pattern orientation; nor are the effects consistent between the eyes of the same observers. In some cases, nasal-temporal differences, though small, are reversed or absent in the other eye. Thus, it seems reasonable to conclude that the results of these earlier studies are due to nasal-temporal differences between observers rather than to hemispheric effects (see also Rijsdijk et al, 1980).

It should be noted that the conclusions of the present study are based on grating patterns that are within the intermediate-to-high spatial-frequency range. Although the oblique effect is not present in the low-spatialfrequency range (Berkley et al., 1975), perhaps laterality differences in contrast sensitivity are revealed at low spatial frequencies. Blake and Mills (1979), however, did not show any hemispheric or hemiretinal effects in contrast sensitivity within this range. In addition, we also failed to demonstrate laterality differences in contrast sensitivity for flickering low-spatial-frequency gratings using either a pattern or a flicker detection criterion. These results are consistent with other research which has failed to account for laterality effects in terms of hemispheric differences in the sensitivity of spatial-frequency channels. For example, Rose (1983) showed that the cerebral hemispheres did not differ in their ability to adapt to highcontrast gratings, in the magnitude of apparent contrast, in the magnitude or time-course of threshold elevation due to adaptation, or in apparent contrast after adaptation. It should be noted that Tei and Owen (1980) had earlier reported hemispheric differences in adaptation to contrast. Rose (1983) has suggested that rather than reflecting differences in sensitivity between the hemispheres, Tei and Owen's (1980) results may reflect information processing asymmetries at a higher level of processing. 
Laterality effects at this level may reflect a limited capacity for handling information or for attending (Moskovitch \& Klein, 1980). In Tei and Owen's (1980) study, observers had to detect activity in two different orientation channels, which is not a simple detection task but one that requires lengthy computations by the visual system. Rose (1983) points out that this task increases the processing load on the system and that it may be only under such conditions that laterality effects are found.

In summary, the present study indicates that the cerebral hemispheres do not differ in either their sensitivity to contrast or in the magnitude of the oblique effect. These results do not necessarily rule out the role of spatialfrequency channels in hemispheric differences, but may indicate that differences between the hemispheres are revealed only in tasks requiring a comparison of stimuli, such as face recognition, but not in simple detection tasks (see also a review by Sergent, 1983b).

\section{REFERENCES}

APPELle, S. (1972). Perception and discrimination as a function of stimulus orientation: The "oblique effect" in man and animals. Psychological Bulletin, 78, 266-278.

ANDREws, D. P. (1967). Perception of contour orientation in the central fovea. I. Short lines. Vision Research, 7, 975-977.

AtKInson, J., \& EGETH, H. (1972). Right hemisphere superiority in visual orientation. Canadian Journal of Psychology, 27, 152-158.

Beaton, A., \& Blakemore, C. (1981). Orientation selectivity of the human visual system as a function of retinal eccentricity and visual hemifield. Perception, 10, 273-282.

Berkley, M. A., KrTterle, F. L., \& WAtkins, D. W. (1975). Grating visibility as a function of orientation and retinal eccentricity. $\mathrm{Vi}$ sion Research, 15, 239-244.

Blake, R., \& MlLL, J. (1979). Pattern and flicker detection examined in terms of the nasal-temporal division of the retina. Perception, 8 , 549-555.

CAmpBell, F. W., Robson, J. G. (1968). Application of Fourier analysis to the visibility of gratings. Joumal of Physiology, 197, 551-566.

DAvson, H. (1972). The physiology of the eye (pp. 481-482). New York: Academic Press.

DiLollo, V. (1981). Hemispheric symmetry in the duration of visible persistence. Perception \& Psychophysics, 29, 21-25.

EgETH, H., EPstein, J. (1972). Differential specialization of the cerebral hemispheres for the perception of sameness and difference. Perception \& Psychophysics, 12, 218-220.

Fairweather, H., Brizzolara, D., Tabossi, P., \& Umiltá, C. (1982). Functional cerebral lateralization: Dichotomy or plurality? Cortex, 18, 51-66.

Fontenot, D. J., \& Benton, A. L. (1972). Perception of direction in the right and left visual fields. Neuropsychologia, 10, 447-452.

GefFen, G., Bradshaw, J. L., \& NetTleton, N. (1972). Hemispheric asymmetry: Verbal and spatial encoding of visual stimuli. In N. Coltheart (Ed.), Readings in cognitive psychology. Toronto: Holt.

Gilbert, C., BAKan, P. (1973). Visual asymmetry in perception of faces. Neuropsychologia, 11, 355-362.

HARMON, L. D. (1973). The recognition of faces. Scientific American, 227, 71-82.

JONES, B. (1980). Sex and handedness as factors in visual-field organization for a categorization task. Journal of Experimental Psychology: Human Performance and Perception, 6, 494-500.

KIMURA, D. (1969). Spatial localization in left and right visual fields. Canadian Journal of Psychology, 23, 445-459.

LoNGDon, K., Ellus, C., IVERSON, S. D. (1976). Hemispheric differ- ences in the discrimination of curvature. Neuropsychologia, 14, 195-202.

Mandelbaum, J., \& Sloan, L. L. (1947). Peripheral visual acuity. American Journal of Ophthalmology, 30, 581-588.

MARKowItZ, H., We WeITZMAN, D. O. (1969). Monocular recognition of letters and Landolt C's in left and right visual hemifields. Joumal of Experimental Psychology, 79, 187-189.

MARZI, C., \& BERLUCCHI, G. (1977). Right visual field superiority for accuracy of recognition of famous faces in normals. Neuropsychologia, 15, 751-756.

MCKINNEY, J. P. (1967). Handedness, eyedness and perceptual stability of the left and right visual fields. Neuropsychologia, 5, 339-344.

MishkIN, M., ForgaYs, D. G. (1952). Word recognition as a function of retinal locus. Joumal of Experimental Psychology, 43, 43-48.

Mitchell, D. E., \& Wilkinson, F. (1974). The effect of early astigmatism on the visual resolution of gratings. Joumal of Physiology, 243, 739-756.

Moscovitch, M. (1979). Information processing in the cerebral hemispheres. In M. S. Gazzaniga (Ed.), Handbook of behavioral neurobiology: Vol. 2, Neuropsychology. New York: Plenum.

Moscovitch, M., \& KLEIN, D. (1980). Perceptual interference for visual words and faces: Implications for models of capacity limitations, attention, and laterality. Journal of Experimental Psychology: Human Performance and Perception, 6, 401-416.

OLDFIELD, R. C. (1971). The assessment and analysis of handedness: The Edinburgh Inventory. Neuropsychologia, 9, 97-113.

OVerton, W., \& WIENER, M. (1966). Visual field position and word recognition threshold. Journal of Experimental Psychology, 71, 249-253.

Patterson, K., \& Bradshaw, J. L. (1975). Differential hemispheric mediation of nonverbal visual stimuli. Joumal of Experimental Psychology: Human Perception and Performance, 1, 246-252.

Posner, M. I., \& Mitchell, R. F. (1967). Chronometric analysis of classification. Psychological Review, 74, 392-409.

RAo, S. M., Rourke, D., WhitMan, R. D. (1981). Spatio-temporal discrimination of frequency in the right and left visual fields: A preliminary report. Perceptual and Motor Skills, 53, 311-316.

RIJsdiJK, J. P., KroON, J. N., \& VAN DER WILDT, G. J. (1980). Contrast sensitivity as a function of position on the retina. Vision Research, 20, 235-241.

RosE, D. (1983). An investigation into hemisphere differences in adaptation to contrast. Perception \& Psychophysics, 34, 89-95.

Rovamo, J., VIRSU, V. (1979). An estimation and application of the human cortical magnification factor. Experimental Brain Research, 37, 495-510.

SERGENT, J. (1982a). The cerebral balance of power: Confrontation or cooperation? Joumal of Experimental Psychology: Human performance and perception, 8, 253-272.

SERGENT, J. (1982b). Influence of luminance on hemispheric processing. Bulletin of the Psychonomic Society, 20, 221-223.

SERGENT, J. (1982c). Theoretical and methodological consequences of variations in exposure duration in visual laterality studies. Perception \& Psychophysics, 31, 451-461.

SERGENT, J. (1983a). The effects of sensory limitations on hemispheric processing. Canadian Journal of Psychology, 37, 345-366.

SERGENT, J. (1983b). Role of the input in visual hemispheric asymmetries. Psychological Bulletin, 93, 481-512.

TEI, B. E., \& OWEN, D. H. (1980). Laterality differences in sensitivity to line orientation as a function of adaptation duration. Perception \& Psychophysics, 28, 479-483.

Teiger, T., GANz, L. (1979). Recognition of faces in the presence of two-dimensional sinusoidal masks. Perception \& Psychophysics, 26, 163-167.

Umita, C., Rizzolattei, G., Marzi, C. A., Zamboni, G., Franzini, C., CAmarda, R., Berlucchi, A. (1974). Hemispheric differences in the discrimination of line orientation. Neuropsychologia, 12 , 165-174.

(Manuscript received October 22, 1984; accepted for publication February 11, 1985.) 